\title{
Hereditary breast and ovarian cancer: Referral source for genetic assessment and communication regarding assessment with nongenetic clinicians in the community setting
}

\author{
Debra Morgan, CCRC $C^{1}$, Heather Sylvester, $M S^{2}$, Frances Lee Lucas, PhD ${ }^{3}$, and Susan Miesfeldt, MD ${ }^{1,2}$
}

Purpose: To examine referral source to cancer genetic services; communication of results of genetic evaluation to clinicians; role of clinicians in postcounseling management; and use of alternative information sources after cancer genetic risk assessment/counseling in the community setting. Methods: Retrospective telephone survey. Setting: A community/private hospital-based cancer genetic counseling service. Patients: Women, at least 21 years of age, who had undergone cancer genetic counseling with (1) at least a $10 \%$ predicted likelihood of carrying a $B R C A 1 / 2$ mutation or (2) a documented BRCA1/2 mutation. Intervention: A 121-item telephone survey. Main outcome measure: (1) initial referral source to cancer genetic services; (2) women's communication of results of cancer genetic assessment to primary and (nongenetic) specialist clinician(s); (3) education and support role played by subjects' physician(s); and (4) use of other hereditary breast and ovarian cancer (HBOC) information resources. Results: Of 225 women eligible for study, 69 (31\%) completed the survey. Sixty-two percent were referred by their medical oncologist; $13 \%$ by their primary care physician, and fewer by their surgeon (6\%) or gynecologist $(4 \%)$. Results of the cancer genetic assessment were not shared with $19 \%$ of primary care clinicians, $26 \%$ of primary gynecologists, $12 \%$ of oncologists, and $36 \%$ of surgeons. Twenty-six percent of participants noted that their primary care clinician had not been involved in their HBOCrelated, cancer prevention decisions, $16 \%$ had not included their gynecologist, $2 \%$ had not involved their oncologist, and $20 \%$ replied that their surgeon had not been involved in these decisions. Overall, clinicians were perceived as supportive when it came to a participants' information and decision support needs. One exception was that $21 \%$ of respondents reported the use by clinicians of medical terms, without definition. Over two-thirds had sought alternative "self-help" HBOCrelated materials, most Internet based. Conclusions: These results have implications for interdisciplinary communication and decision support for those with or at risk for HBOC, cared for in the community setting. Genet Med 2010:12(1):25-31.

Key Words: hereditary breast and ovarian cancer, genetic counseling, community setting, communication, information sources

From the ${ }^{1}$ Maine Medical Center Research Institute, Scarborough; ${ }^{2}$ Maine Center for Cancer Medicine and Blood Disorders, Scarborough; and ${ }^{3}$ Center for Outcomes Research and Evaluation, Maine Medical Center, Portland, Maine.

Susan Miesfeldt, MD, Maine Center for Cancer Medicine and Blood Disorders, 100 U.S. Route One, Scarborough, ME 04074. E-mail: miesfs@mccm.org.

Disclosure: The authors declare no conflict of interest

Submitted for publication February 25, 2009.

Accepted for publication October 14, 2009.

Published online ahead of print December 18, 2009.

DOI: $10.1097 /$ GIM.0b013e3181c60955
$\mathrm{H}^{\mathrm{s}}$ ereditary breast and ovarian cancer (HBOC) risk has important implications for an affected woman's health. Those with known alterations in $B R C A 1$ or $B R C A 2$ are faced with greatly elevated risks for breast cancer, ovarian cancer, and a number of additional malignancies. They are also at risk to develop cancer decades earlier than the general population. A meta-analysis revealed a mean cumulative risk for breast cancer at age 70 years of $57 \%$ (confidence interval [CI]: 47-66\%) and 49\% (CI: $40-57 \%$ ) for $B R C A 1$ and $B R C A 2$ carriers, respectively. Average cumulative risk for ovarian cancer at age 70 years was $40 \%$ (CI: $35-46 \%$ ) for $B R C A 1$ carriers and $18 \%$ (CI: $13-23 \%$ ) for $B R C A 2$ carriers. ${ }^{1}$

Although there have been several studies addressing post genetic counseling management decisions among those with or at significant risk for $\mathrm{HBOC}$ who have undergone cancer genetic counseling and testing in the academic setting, ${ }^{2-9}$ there are sparse data addressing management decisions made by those with a wide range of risk factors for HBOC who have undergone genetic risk assessment and testing through communitybased practices or institutions. Furthermore, few studies have addressed barriers and facilitators to care among women who have undergone risk assessment and genetic testing in the nonacademic setting. An examination of these issues in the community setting is important as increasingly it is likely that cancer genetic risk assessment services will be provided through a range of diverse community systems where one would anticipate that counseling, care, and support resources are more limited and barriers to novel management options more significant.

It is well recognized that primary clinicians play a major role in compliance with established, routine cancer screening and prevention interventions among the general risk population. Specifically, physician recommendation is the most important factor in individuals complying with a range of cancer early detection services, to include regular breast and colon cancer screening. ${ }^{10,11}$ There are limited data concerning the influence of a woman's primary clinician or nongenetic specialist physician on the management of those with or at risk for HBOC. ${ }^{12}$ Particularly, little is known about the role of the primary or nongenetic specialist clinician on the initial assessment and ultimate care of those at risk for this disorder. An examination of this issue is of particular importance in diverse community settings where use of cancer genetic testing services is growing. ${ }^{13,14}$

The overall goal of this study, a component of which is reported here, was to examine risk perceptions, management decisions, and perceived barriers to care among women with diverse risk factors for $\mathrm{HBOC}$ who completed cancer genetic risk assessment (with or without genetic testing) in the community setting. The data presented below relate to the influence of nongenetic clinicians (both primary and specialist) on referral to cancer genetic services, communication of results of the genetic 
evaluation to clinicians, and the role of nongenetic clinicians in cancer risk management decision support.

\section{MATERIALS AND METHODS}

The Institutional Review Board at Maine Medical Center approved all components of this work for subjects recruited through Maine Medical Center and through the Maine Center for Cancer Medicine.

\section{Setting}

This study was done through the Cancer Risk and Prevention Clinic at the Maine Center for Cancer Medicine and Blood Disorders and Maine Medical Center. The Maine Center for Cancer Medicine and Blood Disorders is a private practice Hematology and Oncology group located in Scarborough, ME. Maine Medical Center is a nonprofit primary and tertiary hospital serving Southern Maine. Although affiliated with two New England universities, Maine Medical Center does not support its own allopathic medical school.

\section{Participants}

Eligible participants included women, aged 21 years or older, at risk for or with $\mathrm{HBOC}$, who had completed cancer genetic counseling through the Cancer Risk and Prevention Clinic at the Maine Center for Cancer Medicine and Blood Disorders and Maine Medical Center between June 1998 and May 2005. Women were determined to be at risk for or with HBOC if they had (1) at least a $10 \%$ predicted likelihood of carrying a $B R C A 1$ or $B R C A 2$ mutation based on established computer-based risk assessment models ${ }^{15,16}$ and were untested (BRCA1/2); (2) at least a $10 \%$ predicted likelihood of carrying a $B R C A 1$ or $B R C A 2$ mutation with uninformative $B R C A 1 / 2$ test results (no mutation detected or variant of unknown significance) or; (3) a documented $B R C A 1 / 2$ mutation. At-risk women with informative, "true negative" $B R C A 1 / 2$ test results (noncarriers of an alteration previously identified in the family) were not studied. Furthermore, women with documented mutations in other breast cancer-related genes were not included. This work involved cancer-affected and cancer-unaffected women.

All potential participants had completed cancer genetic counseling under the care of the clinic's geneticist or genetic counselor and its medical oncologist. Through this service, they received information and counseling concerning the medical and genetic implications of HBOC risk and the range of cancer early detection and risk-reducing options available to them, based on their level of risk.

The cancer prevention and early detection strategies discussed with women included intensive screening, chemoprevention, and risk-reducing surgery. All recommendations offered to patients throughout this period were based on the published clinical guidelines available at the time of counseling. ${ }^{17,18}$ Furthermore, women were counseled regarding the process, risks, benefits, and limitations of clinical BRCA1/2 analysis. Based on this information, women elected whether to undergo genetic testing. Among those tested, the cost of this analysis was either billed to the patient's insurance company or paid for by the patient. In a few cases, needs-based $B R C A 1 / 2$ analysis was provided at no cost to the patient by the testing laboratory. Initial testing of the most informative (cancer affected) relative was considered and discussed in all situations where the participant did not have a personal history of cancer. In cases where a cancer-affected relative was unavailable for testing, BRCA1/2 analysis was provided to cancer-unaffected participants.
After their counseling visit(s), women received a standard letter summarizing the information discussed during the session(s) to include the screening and prevention options available to them. A written summary of genetic test results and the implications of these results were sent to those women who underwent $B R C A 1 / 2$ analysis.

Summary results of the counseling session(s) were sent to the woman's referring clinician(s). Records were sent to additional (nonreferring) clinicians, as identified by the patient, and at the request of the patient. The information sent to clinicians included formal genetic test results (among those who elected testing) and a detailed summary of the screening and prevention interventions discussed with the patient at the time of her visit, to include reference to published established guidelines. ${ }^{17,18}$

Women who declined genetic testing were seen on average one time by clinic staff. Those who proceeded to testing generally returned for two additional visits: the first for the DNA testing informed consent process and blood draw and the second for disclosure of test results.

\section{Procedures}

A list of potential participants, meeting the eligibility criteria outlined above, was generated from the clinic's database. All women eligible for study initially received a letter from clinic staff inviting them to participate. The letter fully outlined the goals and processes of the study. Women were asked to return a stamped, addressed postcard stating their interest (or lack thereof) in being involved in this work. Women who declined study participation were not contacted again. We attempted telephone contact on three separate occasions, at different times of the day, with those women who failed to return the study postcard. Those participants who could not be contacted were considered nonresponders. Those who agreed to participate received a phone call from the study's research assistant (D.M.) at which time the study procedure was again reviewed, questions were addressed, verbal informed consent was obtained, and a telephone interview time was scheduled. The interviews were arranged at a time that was convenient to the participant and were designed to last up to 1 hour. All interviews were done in late 2007. Women were offered a \$20 stipend for their participation.

\section{Survey instrument}

The survey was composed of 121 multiple-choice questions. Women were allowed to reply "not applicable" to items that did not relate to their individual management or care. Questions were largely drawn from previously validated instruments to include a survey developed at Creighton University in Omaha, NE (used by permission from Ellen Gritz, PhD, Susan Tinley, and Lari Wenzel, PhD). ${ }^{2}$

The questionnaire was augmented through the addition of 34 new items developed by the study team to specifically address the goals of this study. Added questions included demographic items and items related to precounseling and postcounseling medical management decisions.

The instrument was pilot tested among 10 patients with breast cancer, presenting to Maine Center for Cancer Medicine for medical care. Changes were made to the instrument based on feedback from these women.

The data presented here relate to participants' (1) referral sources; (2) communication of cancer genetic information to clinicians; (3) attitudes regarding the involvement of their clinician(s) in their high-risk care; and (4) use of "self-help" resources. Additional data collected as a result of this work as it related to participants' reasons for undergoing cancer genetic 
counseling, perceptions of cancer risk for self, perceptions regarding barriers to care, and self-reported cancer screening and prevention behaviors will be reported elsewhere.

\section{RESULTS}

\section{Study participants}

A total of 292 women, aged 21 years and older, were seen in the Clinic for assessment of risk for hereditary breast and ovarian cancer between June 1998 and May 2005. Of the 292 patients seen, 34 were either deceased $(n=11)$ or ineligible for study because they had less than a $10 \%$ predicted likelihood of carrying a $B R C A 1$ or $B R C A 2$ mutation based on established risk assessment models. Contact information was not available for an additional 33 potential participants. Of the remaining 225 women, eligible for study, 69 (31\%) completed the telephone survey.

All the women who participated in this work were white. A total of $10(14 \%)$ were of Ashkenazi Jewish descent. Additional demographic and HBOC-related clinical characteristics of the study population are summarized in Table 1 . As noted, a total of 49 participants reported a personal history of breast cancer, whereas none of the women surveyed had a personal history of ovarian cancer. A total of 43 women $(62 \%)$ had undergone $B R C A 1 / 2$ testing, and of these, $16 \%(n=7)$ reported that a

Table 1 Baseline demographic and clinical characteristics $(n=69)$

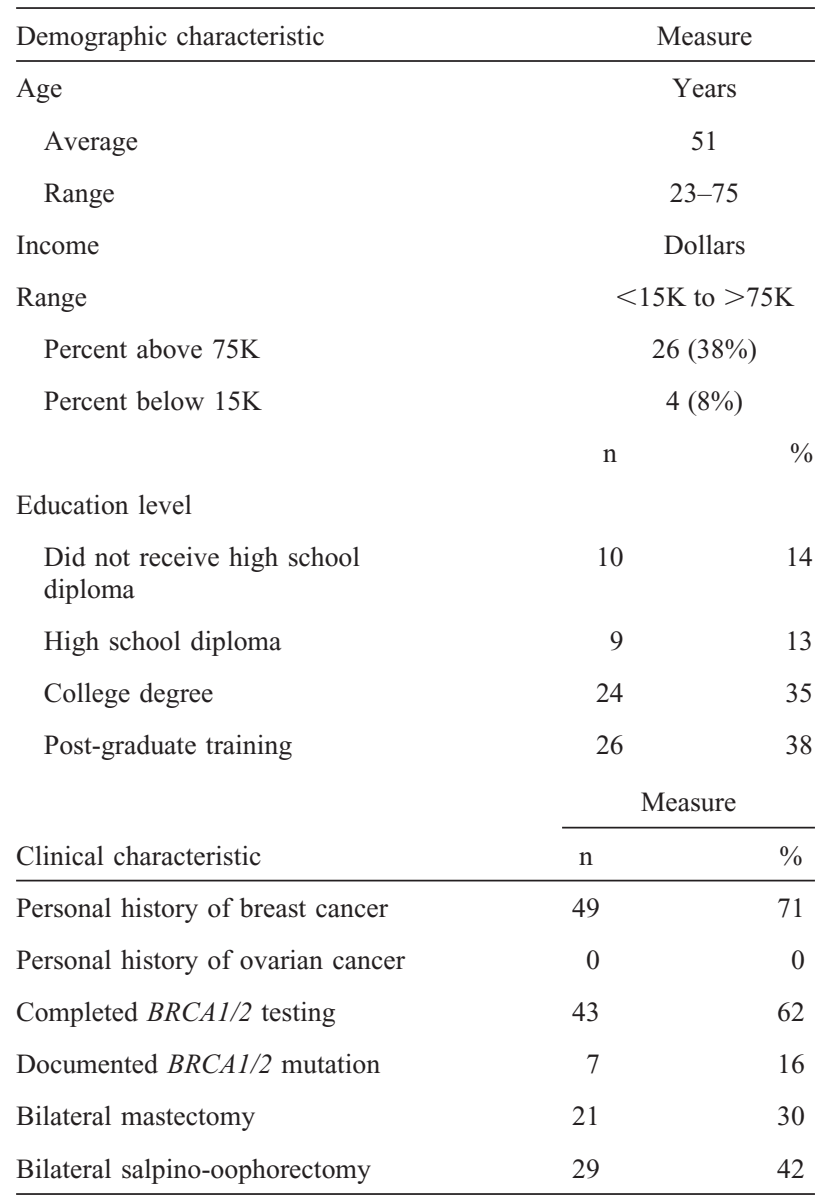

mutation had been detected. Five of the seven documented mutation carriers in this study had a personal history of breast cancer. Among those women with documented mutations, five had undergone bilateral mastectomy for risk reduction or treatment, whereas all seven had undergone bilateral salpingo-oophorectomy prophylactically or for "other reasons."

Of the 62 women without a documented BRCA1/2 mutation, pretest likelihood of carrying a mutation, based on established models, was as follows: $10-29 \%$ in $38(61 \%) ; 30-59 \%$ in 16 $(26 \%)$; and $60 \%$ or greater in $8(13 \%)$. Among these 62 women, $16(26 \%)$ had undergone bilateral mastectomy for treatment $(n=8)$ and/or risk reduction $(n=8)$, whereas $20(35 \%)$ had undergone bilateral salpingo-oophorectomy for risk reduction $(n=14)$ or for "other reasons" $(n=6)$.

\section{Referral sources}

Participants were asked to recall who had referred them for cancer genetic risk assessment and counseling. As noted in Table 2, the majority of women reported being referred by their oncologist $(62 \%)$, with their primary care clinician serving as the second most common source of referral (13\%). A total of 10 $(14 \%)$ women reported that they were "self-referred" or referred from "other sources," to include at the advice of family members $(n=2)$ or other clinicians. As shown, referral source appeared to be influenced by cancer status.

\section{Communication of cancer genetic information to clinician(s)}

Table 3 summarizes participants' communication of the results of their cancer genetic risk assessment with a range of clinicians. Not all women were being followed by each of the

\begin{tabular}{lccc}
\hline Table 2 & Referral source $^{a}(n=69)$ & \\
\hline $\begin{array}{l}\text { Referral } \\
\text { source }\end{array}$ & $\begin{array}{c}\text { Total } \\
\text { population } \\
(n=69)\end{array}$ & $\begin{array}{c}\text { Breast cancer } \\
\text { affected } \\
(n=49)\end{array}$ & $\begin{array}{c}\text { Breast cancer } \\
\text { unaffected } \\
(n=20)\end{array}$ \\
\hline PCP & $9(13)$ & $1(2)$ & $8(40)$ \\
Gynecologist & $3(4)$ & 0 & $3(15)$ \\
Oncologist & $43(62)$ & $41(84)$ & $2(10)$ \\
Surgeon & $4(6)$ & $2(4)$ & $2(10)$ \\
Self & $5(7)$ & $2(4)$ & $3(15)$ \\
Other & $5(7)$ & $3(6)$ & $2(10)$ \\
\hline
\end{tabular}

${ }^{a}$ Data are expressed as $n(\%)$.

PCP, primary care clinician.

Table 3 Communication with clinicians ${ }^{a}(n=69)$

\begin{tabular}{lccc}
\hline Clinician & Not at all & Somewhat & A lot \\
\hline PCP $(n=68)$ & $13(19)$ & $19(28)$ & $36(53)$ \\
Gynecologist $(n=42)$ & $11(26)$ & $9(21)$ & $22(52)$ \\
Oncologist $(n=50)$ & $6(12)$ & $8(16)$ & $36(72)$ \\
Surgeon $(n=53)$ & $19(36)$ & $14(26)$ & $20(38)$
\end{tabular}

$n$ varies for each clinician group because of differing numbers of "not applicable" responses.

${ }^{a}$ Data are expressed as $n(\%)$

$\mathrm{PCP}$, primary care clinician. 
Table 4 Communication with clinicians among those with $B R C A$ mutations $(n=7)$

\begin{tabular}{llllc}
\hline & PCP & Oncologist & Surgeon & Gynecologist \\
\hline Patient 1 & A lot & N/A & A lot & N/A \\
Patient 2 & A lot & A lot & A lot & A lot \\
Patient 3 & A lot & A lot & A lot & A lot \\
Patient $4^{a}$ & A lot & A lot & N/A & A lot \\
Patient 5 & Somewhat & N/A & A lot & A lot \\
Patient 6 & N/A & A lot & A lot & A lot \\
Patient $7^{a}$ & Not at all & Not at all & A lot & N/A
\end{tabular}

${ }^{a}$ History of breast cancer.

N/A, not applicable; PCP, primary care clinician.

disciplines listed in Table 3. The data presented reflect only the subjects being followed by these individual clinical disciplines. Of the 69 women studied, all but one were receiving care from their primary care clinician; 42 were being followed by gynecology; 50 by oncology; and 53 by surgery. As noted in Table 3 , although the majority of women communicated the results of their genetic assessment to several of the clinicians providing their ongoing care, a significant percentage of participants reported that they had not provided any of this risk-related information to one or more of their health care providers. Specifically, 13 of $68(19 \%)$ of respondents reported that they had not shared the results of their cancer genetic evaluation with their primary care clinician; 11 of the $42(26 \%)$ stated that they had not reported this information to their primary gynecologist; 6 of $50(12 \%)$ women followed up by oncology stated that they had not communicated this information to this clinician; and 19 of $53(36 \%)$ women followed up by surgery had not shared the genetic records with this physician. As summarized in Table 4, communication with a range of clinicians was more consistent among the seven women with known $B R C A$ mutations.

A total of nine respondents reported that they had shared the results of the cancer genetic risk assessment with other clinicians to include practitioners from the following disciplines: plastic surgery, ophthalmology, radiology, dermatology, and other genetic professionals.

\section{Perceptions regarding impact of health care provider(s) on high-risk care}

Participants were questioned regarding their health care providers' impact on their personal cancer risk-management decisions. As outlined in Table 5, areas probed among the women surveyed included (1) personal feelings regarding their clinicians' participation in their cancer risk-related management decisions; (2) perceptions related to clinicians' knowledge of HBOC-related cancer-risk management; and (3) feelings concerning their clinician(s)' level of support regarding their disease risk and information needs.

\section{Other sources of information}

Women were asked whether they had sought alternative "self-help" materials related to their risk for HBOC and their risk-related management options. A total of 48 women $(70 \%)$ reported that they had sought additional sources of information after their cancer genetic visit. These sources of information were diverse and included the internet $(n=42)$, the library $(n=$
21), the American Cancer Society, other community resources, conferences, cancer survivor groups, newsletters, friends, journals, magazines, breast cancer coalition, networking, and family members.

\section{DISCUSSION}

Expanding access to molecular technology that allows identification of individuals at significant risk for hereditary breast cancer, particularly $\mathrm{HBOC}$, strengthens the need to further examine the short and long-term impact of cancer genetic risk assessment on those predisposed to this disorder and on their clinicians. Specifically, there is a need to study the potential barriers and facilitators to care among at-risk women from a range of clinical settings and the role(s) that primary and nongenetic specialist clinicians play in this respect.

A central issue associated with potential barriers to high-risk care in the nonacademic setting relates to the initial identification of risk for HBOC in an individual or family and appropriate referral for cancer genetic risk assessment, counseling, and testing services. Equally important is the implementation of risk-based care after the cancer genetic risk assessment process. Presumably, the provision of appropriate care after the cancer genetic risk assessment process is based on effective communication between the at-risk woman and her primary and specialist clinician(s). With these issues in mind, we examined among a population of women with or at risk for HBOC: (1) initial referral sources; (2) communication of the results of the cancer genetic evaluation to primary and (nongenetic) specialist clinician(s); and (3) the education and decision-support role played by participants' nongenetic physician(s). Furthermore, in an effort to examine alternative sources of educational materials and decision support resources, this study began to address use of "self-help" materials associated with HBOC risk and risk management among women counseled in the community setting.

In terms of initial referral for cancer genetic risk assessment and counseling services, the majority of participants reported that their medical oncologist had facilitated this referral. The second most common source of referral was the woman's primary care clinician. Fewer reported referral by their surgeon $(6 \%)$ or primary gynecologist. As expected, referral source varied based on the participant's medical history. As the majority of participants reported a history of breast cancer, it was anticipated at the outset that oncologists would serve as a major source of referral. Increasingly, medical oncologists are becoming involved in accessing and addressing risk for hereditary cancer susceptibility. Along these lines, the American Society of Clinical Oncology has invested significant resources in training oncologists in this area. Competence in cancer genetic risk assessment ${ }^{19-22}$ and counseling has become a prerequisite for medical oncology training programs. ${ }^{23}$ That fewer women reported referral by their surgeon or primary gynecologist is probably largely reflective of the fact that all participants had undergone counseling between 1998 and 2005, when the benefits of risk-reducing surgery were not fully recognized. Furthermore, it is expected that the results noted for gynecologists reflected that none of the study participants had a personal history of ovarian cancer. It is anticipated that recent advances in knowledge regarding the benefits of risk-reducing surgery among those with or at risk for HBOC are influencing more contemporary referral patterns and that today, increasing numbers of at-risk individuals are being referred by a broader range of clinicians. Nevertheless, these data highlight the need for further study in this area and improved systems to identify 
Table 5 Perceptions regarding clinician(s) impact on and knowledge of cancer risk-related care ${ }^{a}(n=69)$

\begin{tabular}{|c|c|c|c|c|c|}
\hline & $\begin{array}{l}\text { Strongly } \\
\text { agree }\end{array}$ & Agree & Neutral & Disagree & $\begin{array}{l}\text { Strongly } \\
\text { disagree }\end{array}$ \\
\hline \multicolumn{6}{|l|}{$\begin{array}{l}\text { Clinicians' participation in cancer risk-related } \\
\text { management decisions }\end{array}$} \\
\hline $\begin{array}{l}\text { My PCP is very involved in my cancer prevention } \\
\text { decisions }(n=69)\end{array}$ & $12(17)$ & 27 (39) & $12(17)$ & $17(25)$ & $1(1)$ \\
\hline $\begin{array}{l}\text { My gyn is very involved in my cancer prevention } \\
\text { decisions }(n=37)\end{array}$ & $8(22)$ & $19(51)$ & $4(11)$ & $6(16)$ & - \\
\hline $\begin{array}{l}\text { My oncologist is very involved in my cancer } \\
\text { prevention decisions }(n=51)\end{array}$ & $19(37)$ & $27(53)$ & $4(8)$ & $1(2)$ & - \\
\hline $\begin{array}{l}\text { My surgeon is very involved in my cancer } \\
\text { prevention decisions }(n=50)\end{array}$ & $16(32)$ & $21(42)$ & $3(6)$ & $8(16)$ & $2(4)$ \\
\hline \multicolumn{6}{|l|}{$\begin{array}{l}\text { Clinicians' knowledge regarding cancer risk-related } \\
\text { management }\end{array}$} \\
\hline $\begin{array}{l}\text { My PCP is not knowledgeable about my cancer } \\
\text { prevention decisions }(n=69)\end{array}$ & $1(1)$ & $5(7)$ & $5(7)$ & $41(59)$ & $17(25)$ \\
\hline $\begin{array}{l}\text { My gyn is not knowledgeable about my cancer } \\
\text { prevention decisions }(n=36)\end{array}$ & - & $2(6)$ & $4(11)$ & $16(44)$ & $14(39)$ \\
\hline $\begin{array}{l}\text { My oncologist is not knowledgeable about my } \\
\text { cancer prevention decisions }(n=48)\end{array}$ & - & $1(2)$ & $1(2)$ & $21(44)$ & $25(52)$ \\
\hline $\begin{array}{l}\text { My surgeon is not knowledgeable about my } \\
\text { cancer prevention decisions }(n=49)\end{array}$ & - & $1(2)$ & $1(2)$ & $24(49)$ & $23(47)$ \\
\hline \multicolumn{6}{|l|}{ Clinicians' support regarding cancer risk-related care } \\
\hline $\begin{array}{l}\text { The doctors and other health care providers } \\
\text { sometimes ignore what I tell them }\end{array}$ & $3(4)$ & $9(13)$ & $3(4)$ & $33(48)$ & $21(30)$ \\
\hline $\begin{array}{l}\text { The doctors and other health care providers } \\
\text { answer all my questions }\end{array}$ & $20(29)$ & $40(58)$ & $1(1)$ & $5(7)$ & $3(4)$ \\
\hline $\begin{array}{l}\text { The doctors and other health care providers act } \\
\text { like I am wasting their time }\end{array}$ & $5(7)$ & $2(3)$ & $4(6)$ & $31(45)$ & $27(39)$ \\
\hline $\begin{array}{l}\text { I trust that the doctors and other health care } \\
\text { providers have my best interest at heart }\end{array}$ & $27(39)$ & $36(52)$ & $2(3)$ & $3(4)$ & $1(1)$ \\
\hline $\begin{array}{l}\text { Sometimes doctors and other health care providers } \\
\text { use medical terms without explaining what they } \\
\text { mean }\end{array}$ & $1(1)$ & $14(20)$ & $3(4)$ & $34(49)$ & $17(25)$ \\
\hline $\begin{array}{l}\text { The doctors and other health care providers listen } \\
\text { carefully to what I have to say }\end{array}$ & $22(32)$ & $41(59)$ & $1(1)$ & $3(4)$ & $2(3)$ \\
\hline $\begin{array}{l}\text { The doctors and other health care providers show } \\
\text { little concern for me }\end{array}$ & $2(3)$ & $1(1)$ & $1(1)$ & $35(51)$ & $30(43)$ \\
\hline
\end{tabular}

HBOC risk across all disciplines involved in the primary care and cancer-related care of women.

Along these same lines, although the majority of women reported that they had communicated the results of their cancer genetic risk assessment to the clinicians who were providing their ongoing care, this communication was not consistent across all health care providers. As anticipated, among the seven women with documented mutations, communication with clinicians seemed more consistent. Nevertheless, one woman with a known mutation and a personal history of breast cancer noted that she had not shared the results of her cancer genetic assessment with her oncologist or her primary care clinician.
One would expect that decisions regarding the distribution of this information may be strongly influenced by past surgical decisions such that those women who had previously undergone bilateral salpingo-oophorectomy were less likely to provide this information to their primary gynecologist, whereas those who had undergone bilateral mastectomy before cancer genetic counseling were less likely to communicate the results of their risk assessment to their surgeon. Among our study population, 4 of 11 of the women who reported that they had not conveyed this information to their primary gynecologist had undergone bilateral salpingo-oophorectomy before their genetic assessment, whereas 6 of 19 of the women who reported that they had 
not communicated with their surgeon had previously undergone bilateral mastectomy. Our numbers are too small to fully address the influence of past surgical decisions on patterns of communication with clinicians. Furthermore, we did not assess communication of this information between clinicians from different disciplines, practicing in a multidisciplinary setting. These are areas in need of further study.

When women were questioned regarding their clinicians' input in their HBOC-related management decisions, although the majority of women involved most of their clinicians in their prevention decisions, it seemed that this input also varied, based on medical discipline. As noted above, one would expect that the surgery-specific data should be reflective of past surgical decisions, such that those women who had already undergone bilateral salpingo-oophorectomy or bilateral mastectomy did not believe that they needed to include their gynecologist or surgeon in their treatment decisions. Our results did not support this, for among the participants of this study, only two of six who disagreed with the statement "My gyn is very involved in my cancer prevention decisions" had undergone bilateral salpingo-oophorectomy before genetic counseling, whereas none of the 10 women who disagreed with this statement related to their surgeon had previously undergone bilateral mastectomy. It is difficult to make conclusions from these results because of our small sample size. As with communication, there is a need for additional research related to decision support among those with or at risk for HBOC.

Our data related to clinician communication and decision support, although not conclusive, raise concern, for many of the medical management decisions faced by women with or at risk for HBOC involve a wide range of medical disciplines. This is particularly the case for women who are candidates for riskreducing surgery, to include bilateral salpingo-oophorectomy or bilateral mastectomy. Among these women, if results of the cancer genetic risk assessment are not shared with those clinicians involved in risk-reducing surgical decisions, they may not be exposed to the valuable multidisciplinary input of these and other services (i.e., plastic surgery) as they consider the full spectrum of risk-reducing management options available to them, and the risks, benefits, and limitations of these options.

We did not address with subjects factors influencing communication with clinicians, to include a full examination of the impact of past surgical decisions on patterns of communication. Furthermore, we did not completely assess why women had not sought the participation of each of their clinicians in their management decisions. We see this as a limitation of this work. These issues did not seem to relate to women's perceptions of their clinicians' discipline-specific knowledge because overall, participants believed that their primary and specialist clinicians were knowledgeable in this area. Communication of cancer genetic risk-related information to health care providers is an area in need of further study, as the management of women with or at risk for HBOC increasingly involves a team of professionals that includes a uniquely diverse group of clinicians. To fully benefit from a range of opinions, effective communication of valid information from the at-risk individual to her clinicians, as well as between all of her physicians, is essential for the provision of quality and decision-sensitive high-risk care.

Overall, clinicians were perceived as supportive when it came to a participant's information and decision support needs. One exception was that $21 \%$ of respondents found that their clinicians used medical terms without definition. This is an important issue as many of the terms and concepts used in the genetic risk assessment and management processes are quite complex. Furthermore, to date, there have been a limited num- ber of lay-directed educational materials available to individuals and families undergoing cancer genetic risk assessment, counseling, and testing. Although this is beginning to change through the recent availability of resources such as CancerNet, ${ }^{24}$ a void remains in this area.

Importantly, clinicians were not the only source of HBOCrelated information among the women studied. Seventy percent of participants noted that they had sought other sources of information related to $\mathrm{HBOC}$ risk and management of risk. These sources varied widely. As anticipated, the most common source of information was the Internet. This raises concern because of the wide range of quality of Internet-based educational resources in this area. We did not assess the perceived value of the external sources of information used. This is an area in need or further research because increasing numbers of individuals and families seek cancer genetic testing from a wide range of services to include the private practice setting where genetic counseling and genetic educational resources are presumed to be more limited.

There were several important limitations to this work. First, the survey instrument used for this work was pilot tested among a group of patients with breast cancer rather than a group of women who were known to be at risk for HBOC. This group of women may not have met the study's eligibility criteria. We recognize that this may have limited the findings from the pilot test. Second, the study's response rate was low. As a result, our study population was relatively small. The low response rate noted is likely reflective of the fact that we attempted to recruit participants from a patient population dating back to 1998. It is expected that a number of the women seen in the late 1990s to early 2000s had moved or were otherwise not available for study. In addition, the population involved in this work included a geographically and medically diverse group of women being managed in the community setting. Taking into consideration this divergent study population, at the outset of this study, we anticipated a variable and overall low response to our requests to participate in this work. We recognize that both the small size of our study population and the low response rate limit the generalizability of this work. Third, our study involved only those women willing and able to participate in the telephone survey, further limiting the applicability of our findings. Fourth, this study included a cohort of women recruited from a single genetic counseling site. Therefore, our results are largely influenced by the knowledge, attitudes, and beliefs of the genetic professionals providing care to these women. Fifth, although our study population included women from divergent geographic and socioeconomic backgrounds, the ethnic and racial diversity of this population was limited, reflecting the demographics of the state of Maine. Finally, our study population was drawn from a large time frame, during which clinical data were accumulating rapidly regarding the care of those with or at risk for $\mathrm{HBOC}$. We recognize that these accumulating data may have impacted the issues addressed here when comparing those study participants seen earlier in this time frame, versus those seen more recently.

Future work in this area should examine at-risk women's communication of HBOC-risk-related information to a range of clinicians, as well as communication of this information between physicians, among a broader population of at-risk women and families seen through diverse community-based settings, to include those women receiving their counseling and testing services by nongenetic professionals. This work should also examine on a broader scope, the involvement of a range of clinicians in the evaluation, education, support, and care of at-risk women. Data in this area are invaluable to the develop- 
ment of effective risk assessment and decision support interventions within the context of interdisciplinary care for those women with or at risk for $\mathrm{HBOC}$, receiving their care in the community setting.

\section{ACKNOWLEDGMENTS}

This work was supported by the Maine Cancer Foundation and the Maine Medical Center Research Institute. The authors are very grateful to the women who participated in this study.

\section{REFERENCES}

1. Chen S, Parmigiani G. Meta-analysis of BRCA1 and BRCA2 penetrance. $J$ Clin Oncol 2007;10:1329-1333.

2. Tinley ST, Houfek J, Watson P, et al. Screening adherence in BRCA1/2 families is associated with primary physicians' behavior. Am J Med Genet $A$ 2004;125A:5-11.

3. Lerman C, Hughes C, Croyle RT, et al. Prophylactic surgery decisions and surveillance practices one year following BRCA1/2 testing. Prev Med 2000; $31: 75-80$.

4. Botkin JR, Smith KR, Croyle RT, et al. Genetic testing for a BRCA1 mutation: prophylactic surgery and screening behavior in women 2 years post testing. Am J Med Genet A 2003;118A:201-209.

5. Meijers-Heijboer H, Brekelmans CT, Menke-Pluymers M, et al. Use of genetic testing and prophylactic mastectomy and oophorectomy in women with breast or ovarian cancer from families with a BRCA1 or BRCA2 mutation. J Clin Oncol 2003;21:1675-1681.

6. Peshkin BN, Schwartz MD, Isaacs C, Hughes C, Main D, Lerman C. Utilization of breast cancer screening in a clinically based sample of women after BRCA1/2 testing. Cancer Epidemiol Biomarkers Prev 2002;11:11151118

7. Scheuer L, Kauff N, Robson M, et al. Outcome of preventive surgery and screening for breast and ovarian cancer in BRCA mutation carriers. J Clin Oncol 2002;20:1260-1268.

8. Isaacs C, Peshkin BN, Schwartz M, Demarco TA, Main D, Lerman C. Breast and ovarian cancer screening practices in healthy women with a strong family history of breast or ovarian cancer. Breast Cancer Res Treat 2002; $71: 103-112$.

9. Metcalfe KA, Lubinski J, Ghadirian P, et al. Predictors of contralateral prophylactic mastectomy in women with a BRCA1 or BRCA2 mutation: the
Hereditary Breast Cancer Clinical Study Group. J Clin Oncol 2008;26: 1093-1097.

10. McPhee SJ, Detmer WM. Office-based interventions to improve delivery of cancer prevention services by primary care physicians. Cancer 1993; 72(suppl 3):1100-1112.

11. Smith RA, Mettlin CJ, Davis KJ, Eyre H. American Cancer Society guidelines for the early detection of cancer. CA Cancer J Clin 2000;50:34-49.

12. Keating NL, Stoeckert KA, Regan MM, DiGianni L, Garber JE. Physicians' experiences with BRCA1/2 testing in community settings. J Clin Oncol 2008;26:5789-5796.

13. Friedman LC, Cooper HP, Webb JA, Weinberg AD, Plon SE. Primary care physicians' attitudes and practices regarding cancer genetics: a comparison of 2001 with 1996 survey results. $J$ Cancer Educ 2003;18:91-94.

14. Wideroff L, Freedman AN, Olson L, et al. Physician use of genetic testing for cancer susceptibility: results of a national survey. Cancer Epidemiol Biomarkers Prev 2003;12:295-303.

15. Barcenas CH, Hosain GM, Arun B, et al. Assessing BRCA carrier probabilities in extended families. $J$ Clin Oncol 2006;24:354-360.

16. James PA, Doherty R, Harris M, et al. Optimal selection of individuals for BRCA mutation testing: a comparison of available methods. J Clin Oncol 2006;24:707-715

17. Burke W, Daly M, Garber J, et al. Recommendations for follow-up care of individuals with an inherited predisposition to cancer. II. BRCA1 and BRCA2. Cancer Genetics Studies Consortium. JAMA 1997:277:997-1003.

18. National Comprehensive Cancer Network. NCCN Clinical Practice Guidelines in Oncology, 2009. Available at: http://www.nccn.org/professionals/ physician gls/PDF/breast.pdf. Accessed April 15, 2009.

19. Statement of the American Society of Clinical Oncology: genetic testing for cancer susceptibility, Adopted on February 20, 1996. J Clin Oncol 1996; 14:1730-1736.

20. American Society of Clinical Oncology policy statement update: genetic testing for cancer susceptibility. J Clin Oncol 2003;21:2397-2406.

21. American Society of Clinical Oncology. Resource document for curriculum development in cancer genetics education. American Society of Clinical Oncology. J Clin Oncol 1997;15:2157-2169.

22. Offitt K GJ, Grady M, Greene MH, et al. ASCO Curriculum: Cancer Genetics \& Cancer Predisposition testing, 2nd ed. Alexandria, VA: ASCO Publishing, 2004

23. American Board of Internal Medicine. Available at: http://www.abim.org/ certification/policies/imss/medon.aspx. Accessed November 18, 2009.

24. American Society of Clinical Oncology. Cancer Net. Available at: http:// www.cancer.net. Accessed February 12, 2009. 